\title{
Cambio en el patrón de presentación del cáncer de próstata en Oviedo en los últimos 10 años: patrón de presentación, manifestaciones clínicas y anatomo-patológicas (Parte I)
}

\author{
Álvarez-Múgica M, Fernández Gómez JM, Escaf Barmadah S, Jalón Monzón A, \\ González Álvarez RC, Regadera Sejas FJ.
}

Servicio de Urología. Hospital Universitario Central de Asturias. Oviedo.

Actas Urol Esp. 2006;30(10):974-979

\section{RESUMEN}

CAMBIO EN EL PATRÓN DE PRESENTACIÓN DEL CÁNCER DE PRÓSTATA EN OVIEDO EN LOS ÚLTIMOS 10 AÑOS: PATRÓN DE PRESENTACIÓN, MANIFESTACIONES CLÍNICAS Y ANATOMO-PATOLÓGICAS (PARTE I)

Objetivos: Describir el patrón de presentación tanto clínico como anatomo-patológico de los pacientes diagnosticados de cáncer de próstata en nuestro servicio, comparando los datos obtenidos en el año 1995 y en el 2004.

Material y métodos: Se revisaron las 216 historias clínicas de los pacientes diagnosticados en 1995 y 2004, recogiéndose unos datos según un protocolo establecido.

Resultados: La edad media al diagnóstico fue significativamente más baja en el año 2004. En ese año, prevaleció el incremento de la concentración de PSA como causa de diagnóstico, seguido por los síntomas urinarios del tracto urinario inferior (STUI), mientras que en el año 1995 destacaron los STUI y el tacto rectal sospechoso. Se observó una mayor proporción de tumores de alto grado en el año 1995, donde los tumores predominantes fueron los de alto riesgo mientras que en el año 2004 fueron los de bajo riesgo y encontramos una mayor proporción de tumores de bajo grado.

Conclusiones: La migración de la estadificación clínico patológica hacia estadios más precoces descrita tras el uso generalizado del PSA en pacientes asintomáticos, se confirmó en nuestro estudio, como demuestra la mayor proporción de tumores de alto grado con Gleason entre 8-10 ng/ml diagnosticados en la serie del año 1995 y un mayor número de tumores de bajo grado en el año 2004 .

Palabras clave: Adenocarcinoma de próstata. Antígeno prostático específico. Suma de Gleason.

\section{ABSTRACT}

CHANGES IN THE CLINICAL AND ANATOMO-PATHOLOGICAL PRESENTATION OF PROSTATE CANCER IN OVIEDO IN THE LAST 10 YEARS (PART I)

Objectives: To describe the clinical and pathological factors of prostate adenocarcinomas diagnosed in our department in the years 1995 and 2004.

Material and method: We review the 216 patients diagnosed in both years, recording several features.

Results: The mean age was significatively lower in the year 2004. In that year, the increments of the PSA levels was the main reason for the diagnosis of the prostate cancer, followed by low urinary tract symptons (LUTS), while the LUTS was the main reason in 1995. There was a greater proportion of high grade tumors in 1995 and also in this year, high risk tumors were the most frequently found, while in 2004 low risk and low grade tumors were predominant.

Conclusions: Due to the general determinations of PSA levels in asymptomatic patients in 2004, we found a greater proportion of high grade tumors in 1995 and lower grade tumors in 2004 .

Keywords: Prostate adenocarcinoma. Prostate specific antigen. Gleason score. 
$\mathrm{E}^{1}$ cáncer de próstata es el tumor urológico más frecuente, y el tercer tumor más frecuente en varones después del melanoma y del cáncer de pulmón, siendo en Estados Unidos (EEUU) el segundo después del cáncer de piel. En Europa se ha estimado que el cáncer de próstata es el tumor maligno más frecuente que afecta al hombre, siendo la segunda causa de muerte por cáncer, después del pulmonar. En la actualidad, se desconoce la incidencia y prevalencia de la enfermedad en la población española, ya que no existen estudios epidemiológicos en este sentido. No obstante, de los datos epidemiológicos obtenidos de las distintas Comunidades Autónomas, se puede realizar una extrapolación al total de la población española, de forma que la incidencia de cáncer de próstata es de 120,1 casos por 100.000 varones, mientras que a nivel europeo y mundial es de 103,5 y 68,8 casos por 100.000 varones, respectivamente ${ }^{1}$. Cada año se diagnostica un cáncer de próstata por cada de 500.000 varones en todo el mundo y son más de 200.000 los que fallecen a causa de esta enfermedad ${ }^{2}$. En la Unión Europea, la incidencia es de unos 84.000 casos por año y causa 35.000 fallecimientos anuales. En España, en el año 2002, se produjeron 5.678 muertes por esta enfermedad, lo que representa una tasa de 27,6 muertes/ 100.000 varones y año, siendo el tercer tumor maligno en cuanto a mortalidad tras el cáncer de pulmón y el colorrectal. Desde el año 1952 al 1996 la tendencia de mortalidad por este tumor en España ha experimentado un aumento del orden del $1 \%$ anual, disminuyendo ligeramente cuando se limita el análisis al periodo de 1982$1996{ }^{3}$. La Sociedad Americana contra el Cáncer (ACS) estima que, en el año 2004, se diagnosticaron en EEUU 230.900 nuevos casos y unos 29.900 fallecieron por esta entidad. El cáncer de próstata afecta a 1 de cada 6 varones en EEUU. Se ha descrito un aumento en el número de casos diagnosticados, así como una disminución en la tasa de mortalidad, probablemente debido a los programas de cribado de cáncer de próstata que se han puesto en funcionamiento.

Con la incorporación de la determinación de la concentración plasmática del Antígeno Prostático Específico (PSA) en pacientes asintomáticos, desde comienzos de la década de los 90 , se ha producido una migración de la estadificación clínica del cáncer de próstata hacia los estadios más iniciales. Sin embargo, no se ha demostrado aún que estos efectos hayan disminuido la mortalidad por cáncer de próstata, salvo algunas excepciones ${ }^{4}$. Antes de la introducción del PSA, los pacientes no eran tratados hasta que no presentaban una progresión sintomática de la enfermedad. En la actualidad es posible identificar a los pacientes con un cáncer de próstata más precozmente, en base a un incremento de la concentración de PSA, de forma que se les puede aplicar un tratamiento con lo que se les interviene más precozmente. Esto ha originado un aumento en la supervivencia en los últimos 20 años, de tal manera que la supervivencia a 5 años para el conjunto de todos los estadios ha aumentado del $67 \%$ al $98 \%$, alcanzando el $86 \%$ y el $56 \%$ a los 10 y a los 15 años respectivamente ${ }^{5}$.

\section{MATERIAL Y MÉTODOS}

Realizamos un estudio retrospectivo sobre la base de datos de los pacientes diagnosticados de cáncer de próstata en el Servicio de Urología I del Hospital Universitario Central de Asturias (HUCA) en los años 1995 y 2004.

Se han estudiado 203 pacientes de los 216 diagnosticados de adenocarcinoma de próstata en los dos años, 143 corresponden al año 2004 y 60 a 1995.

Se excluyeron del estudio los pacientes que:

- No tenían historia clínica completa.

- Tenían tumor de próstata no adenocarcinoma.

El estudio se basó en datos recogidos en la consulta externa, en su mayoría, evidenciándose una serie de diferencias y variables dependiendo del año en el que se recogieron, ya que en 1995 no se realizaban en el Servicio de Urología ecografias transrectales de próstata, lo que imposibilitaba la realización de biopsias ecodirigidas y la estadificación de un tumor visible en la ecografía como T2. Otra de los variables utilizada en el año 2004 y no en 1995 fue el índice de PSA libre/PSA total, el cual ha permitido tomar decisiones sobre la realización de biopsias prostáticas en el grupo más importante de pacientes, que es aquel en el que los valores de PSA total se encuentra entre 4 y $10 \mathrm{ng} / \mathrm{ml}$. Se revisaron las 
historias clínicas de los pacientes que seleccionamos para el estudio recogiéndose los datos según un protocolo establecido previamente en el que se incluían las siguientes variables.

- Datos de filiación del paciente, que incluyen (nombre y apellidos, número de historia clínica, número de biopsia asignado en Anatomía Patológica, edad al diagnóstico y fecha de diagnóstico).

- Número de morbilidades asociadas.

- Motivo de diagnóstico.

- Tacto rectal.

- Valor del PSA en el momento del diagnóstico.

- Índice del PSA en el momento del diagnóstico.

- Densidad del PSA.

- Valores previos del PSA y fecha de los mismos.

- Velocidad del PSA.

- Meses con niveles elevados del PSA.

- Método de diagnóstico.

- Biopsias prostáticas previas al diagnóstico.

- Presencia de PIN previo.

- Gleason primario del tumor.

- Gleason secundario del tumor.

Se incluyeron como motivo de diagnóstico: LUTS, PSA solicitado por un urólogo, PSA solicitado por el médico de cabecera, el tacto rectal, la presencia de metástasis óseas, la presencia de ureterohidronefrosis uni o bilateral, $\mathrm{u}$ otras causas.

En cuanto a las patologías o morbilidades asociadas, se incluyen las siguientes: SIDA, hepatopatía importante, diabetes con repercusión orgánica, EPOC importante, cardiopatía isquémica, insuficiencia cardiaca congestiva, fallo renal crónico importante, vasculopatía periférica avanzada, ictus con secuelas, tumor con metástasis con menos de 2 años de tratamiento y leucemia o linfoma.

Los resultados fueron almacenados en una base de datos y procesados mediante el programa SPSS versión 11 de aplicaciones estadísticas. Se estableció significación estadística para valores de $\mathrm{p}$ menores de 0,05 con intervalo de confianza del 95\%.

\section{RESULTADOS}

La edad media global de los pacientes en el momento del diagnóstico fue de 70,12 $\pm 8,26$ años (50-92). En el año 1995 fue de 73,03 \pm 9,7 (53-92) años y en 2004 de 68,9 \pm 7,26 (50-84) años, siendo esta última significativamente inferior ( $\mathrm{p}=0,001$ ) que la del año 1995 (Fig. 1).

La distribución por grupos de edad en cada año de diagnóstico se pone de manifiesto en la Tabla 1.

En el año 1995 el mayor porcentaje de pacientes (70\%) diagnosticados de CP correspondían al grupo de más de 70 años mientras que en el año 2004 la mayoría $(72,1 \%)$ se encontraba entre 60 y 75 años.

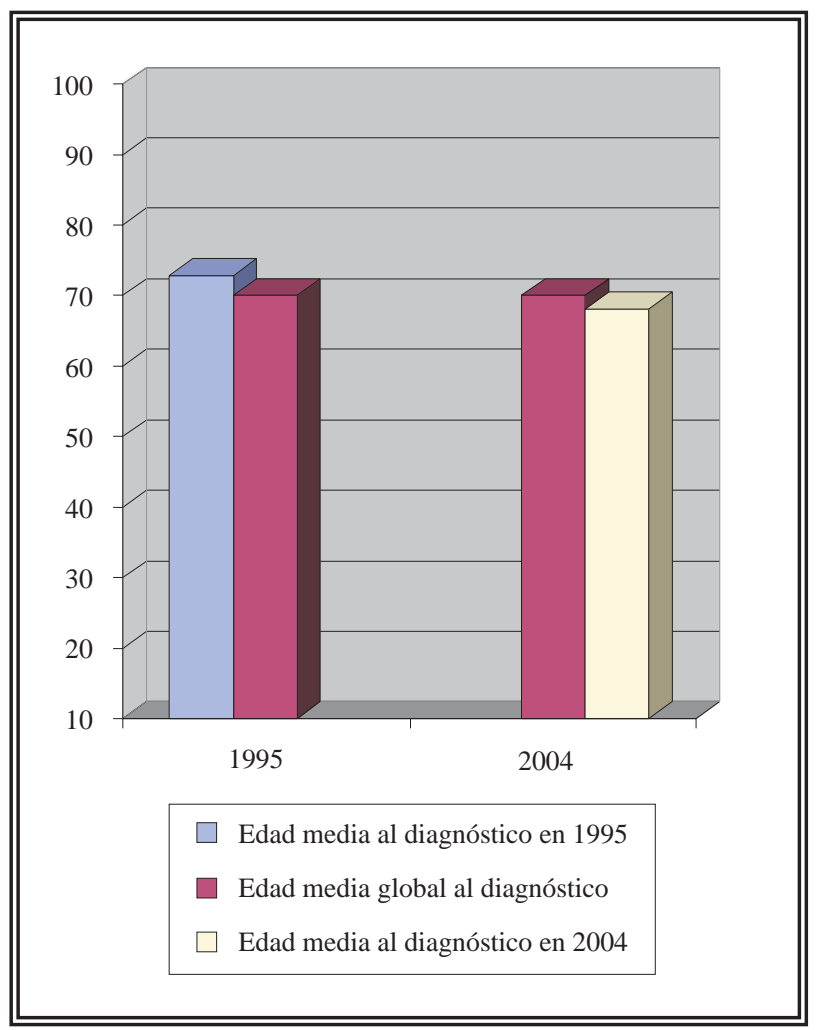

FIGURA 1. Edad media de diagnóstico en ambos grupos.

Tabla 1

Número de casos diagnosticados en 1995 y 2004.

\begin{tabular}{lccc}
\hline $\begin{array}{l}\text { Edad diagnóstico } \\
\text { (en años) }\end{array}$ & $\begin{array}{c}\mathbf{1 9 9 5} \\
\mathbf{n}(\%)\end{array}$ & $\begin{array}{l}\mathbf{2 0 0 4} \\
\mathbf{n}(\%)\end{array}$ & $\begin{array}{l}\text { Total } \\
\mathbf{n}(\%)\end{array}$ \\
\hline $50-59$ & $7(11,7)$ & $15(10,5)$ & $22(10,8)$ \\
$60-69$ & $11(18,3)$ & $58(40,5)$ & $69(34,0)$ \\
$70-75$ & $18(30,0)$ & $45(31,5)$ & $63(31,0)$ \\
$>75$ & $24(40,0)$ & $25(17,5)$ & $49(24,1)$ \\
Total & $60(100,0)$ & $143(100,0)$ & $203(100,0)$ \\
\hline
\end{tabular}


Respecto a las patologías asociadas, encontramos diferencias significativas ( $\mathrm{p}=0,033$ ) entre los pacientes diagnosticados en los años 1995 y 2004. Así, en el año 1995, se encontraron 2 o más patologías asociadas graves en el $15 \%$ de casos, frente a sólo el 5,6\% en el año 2004. La media de edad fue significativamente superior en los pacientes con 2 o más patologías asociadas $(76,7 \pm 8,5$ años) frente a aquellos que presentaban solamente una $(70,4 \pm 6,9$ años) o ninguna comorbilidad $(70,7 \pm 8,2$ años). No se encontraron diferencias significativas en el tipo de procesos entre ambos años, destacando únicamente una mayor proporción de EPOC severo y neoplasias activas intercurrentes en los pacientes con cáncer de próstata en el año 1995 respecto al 2004.

Globalmente, la determinación del PSA fue el motivo más frecuente que indujo a sospechar el tumor $(48,8 \%)$, solicitado por el médico de cabecera $(21,7 \%)$ o por el urólogo $(27,1 \%)$. Este porcentaje se eleva al $63,7 \%$ si sólo se analiza el año 2004 , y sería del $13,4 \%$ si se estudia aisladamente el año 1995, (sólo en el 1,7\% de los casos en este año el PSA lo había solicitado el médico de cabecera). En 64 pacientes (31,5\%) el cáncer de próstata fue diagnosticado durante el seguimiento por síntomas del tracto urinario inferior (STUI), si bien el porcentaje en el año 2004 $(28,7 \%)$ es menor que el conseguido en 1995 (38,3\%) (Fig. 2). De los pacientes controlados por

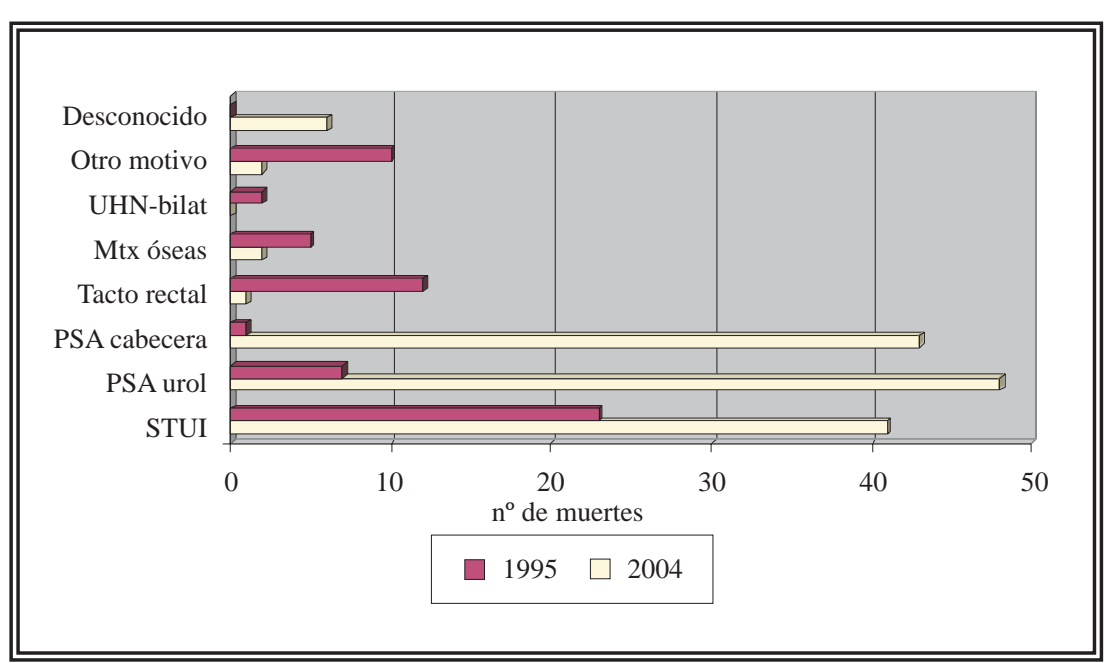

FIGURA 2. Causa de diagnóstico del cáncer de próstata en los años 1995 y 2004 en el Servicio de Urología I del HUCA. (Mtx óseas: Metástasis óseas; PSA urol: PSA urólogo; STUI: Síntomas del tracto urinario inferior; UHN-bilat: Ureterohidronefrosis bilateral).
STUI en 1995, 13 fueron diagnosticados de cáncer de próstata por tacto rectal sospechoso $(56,5 \%)$ y el resto, $(43,5 \%)$, por elevación del PSA. Sin embargo, de los pacientes controlados por STUI en el año 2004, 18 fueron diagnosticados de cáncer de próstata por tacto rectal sospechoso el $(43,9 \%)$ y 23 pacientes por elevación del PSA (56,1\%). Encontramos también diferencias significativas $(p<0,001)$ en las concentraciones del PSA en relación con el año de diagnóstico. Así, la concentración media de PSA en los pacientes diagnosticados en el año 1995 fue de 246,04 \pm $664,3 \mathrm{ng} / \mathrm{ml}$ (1,7-3781), mientras que en los diagnosticados en el año 2004 fue de 15,64 \pm $21,62 \mathrm{ng} / \mathrm{ml}$ (1,5-193). Se encontró una asociación significativa $(\mathrm{p}=0,004)$ entre los grupos de edad y los valores del PSA agrupados en rangos de concentraciones 0-4, 4-10 y $>10 \mathrm{ng} / \mathrm{ml}$, de forma que los pacientes con edad más avanzada tenían valores de PSA más elevados. En este sentido, hallamos una correlación significativa $(\mathrm{p}=0,001)$ entre edad y concentraciones del PSA (indice de Pearson de 0,25). Existieron diferencias significativas $(p=0,022)$ en las concentraciones del PSA entre los pacientes con tacto rectal sospechoso y normal. En este sentido, cuando el tacto fue patológico la concentración media de PSA fue muy superior $(22,64 \mathrm{ng} / \mathrm{ml})$ a la de los pacientes con tacto rectal normal $(14,61 \mathrm{ng} / \mathrm{ml})$. Respecto a las variables evaluadas del PSA, se encontró que la media del índice de PSA libre/total, en pacientes con CP en 2004, era de $13,9 \%$. Al comparar la distribución de los grupos de la suma del score de Gleason con el año del diagnóstico evidenciamos diferencias significativas $(\mathrm{p}=0,023)$, observándose una proporción de tumores con suma de Gleason de 8-10 mayor de la esperada en los pacientes diagnosticados en el año 1995 respecto a los del año 2004, y a la inversa una mayor proporción de tumores de bajo grado correspondientes al año 2004 respecto a los encontrados en el año 1995 (Fig. 3). También se observaron diferencias signifi- 


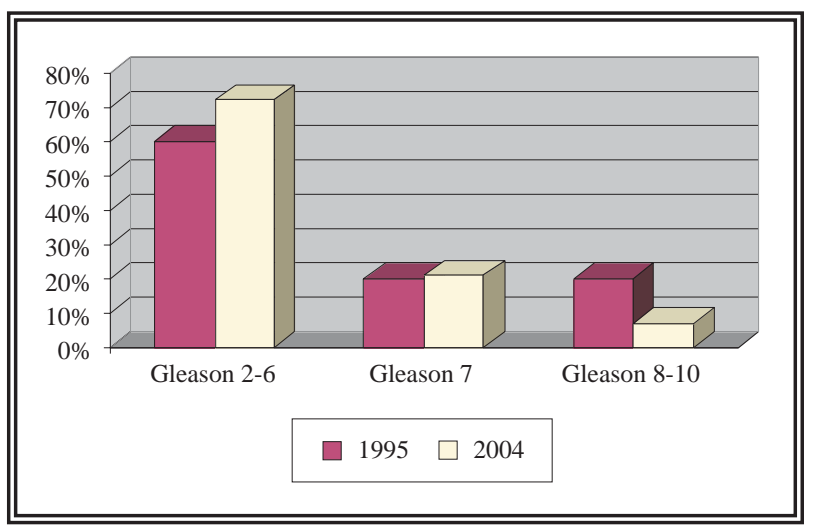

FIGURA 3. Score de Gleason de los pacientes diagnosticados de cáncer de próstata en los años 1995 y 2004.

cativas $(p=0,011)$ en la distribución de Gleason primario (1-3 vs 4-5), que tendió a ser menos agresivo en los tumores diagnosticados en el año 2004.

Globalmente, encontramos diferencias significativas al comparar la suma de Gleason que fue mayor en pacientes con tacto rectal sospechoso y con concentraciones elevadas de PSA respecto a los pacientes en los que el tacto fue normal o tuvieron concentraciones de PSA inferiores a $10 \mathrm{ng} / \mathrm{ml}$ (U de Mann-Whitney).

Atendiendo a los grupos de riesgo establecidos por D'Amico en 1998 para los tumores localizados (no se consideran los metastáticos), encontramos una asociación significativa $(\mathrm{p}<0,001)$ entre los grupos y el año de diagnóstico, evidenciando mayor proporción de la esperada de tumores de alto riesgo en la serie de pacientes del año 1995 y de bajo riesgo en la del año 2004 (Tabla 2).

\section{DISCUSIÓN}

El presente estudio fue diseñado para evaluar y comparar el patrón de presentación, así como las características clínico-patológicas de

\section{Tabla 2}

Agrupación de pacientes según grupos de riesgo establecidos por D’Amico. (Entre paréntesis, porcentajes de los pacientes en cada grupo en relación con el año de diagnóstico).

\begin{tabular}{lcccc}
\hline $\begin{array}{l}\text { Grupos de D'Amico } \\
\text { Año diagnóstico }\end{array}$ & $\begin{array}{c}\text { Bajo riesgo } \\
\text { n (\%) }\end{array}$ & $\begin{array}{c}\text { Medio riesgo } \\
\text { n (\%) }\end{array}$ & $\begin{array}{c}\text { Alto riesgo } \\
\text { n (\%) }\end{array}$ & $\begin{array}{c}\text { Total } \\
\text { n (\%) }\end{array}$ \\
\hline 1995 & $9(18,0)$ & $14(28,0)$ & $27(54,0)$ & $50(100)$ \\
2004 & $58(43,3)$ & $43(32,1)$ & $33(24,6)$ & $134(100)$ \\
Total & $67(36,4)$ & $57(31,0)$ & $60(32,6)$ & $184(100)$ \\
\hline
\end{tabular}

los tumores de próstata diagnosticados en el Servicio de Urología 1 del HUCA en los años 1995 y 2004.

Se observó que, debido a la nueva metodología de estudio y diagnóstico del cáncer de próstata, la edad media de diagnóstico, 68,9 años, fue significativamente menor en el año 2004 que en 1995. El $72,1 \%$ de los pacientes diagnosticados en el año 2004 tenían una edad comprendida entre 60 y 75 años. Estos resultados coinciden con los de otros estudios similares al nuestro y que muestran resultados parecidos respecto a la edad media de los pacientes en el momento de diagnóstico ${ }^{4,6}$. Sin duda, la incorporación de la determinación del PSA en suero, en la década de los 90, en pacientes asintomáticos, ha influido en que se haya conseguido un diagnóstico más precoz de la enfermedad.

Existen diferencias significativas con respecto al año de diagnóstico ( $\mathrm{p}=0,033)$, en el número de patologías asociadas en los pacientes diagnosticados de CP, presentando más de dos patologias el 15\% de los pacientes diagnosticados en el año 1995 frente a sólo el 5,6\% de los diagnosticados en el año 2004. Este hallazgo probablemente esté relacionado con la edad media del diagnóstico ya que en el año 2004 ésta resultó ser significativamente menor que la del año 1995. Herránz Amo et al. ${ }^{6}$, observaron que más del $67 \%$ de los pacientes presentaban patologías asociadas. En nuestra serie estos resultados eran un poco inferiores, $45 \%$ y $40 \%$ presentaban morbilidad asociada en los años 1995 y 2004 respectivamente.

Una de las diferencias más claras entre las dos series de pacientes fue la observada en el motivo de diagnóstico. En el año 2004, el diagnóstico de $\mathrm{CP}$, en el $64 \%$ de los pacientes, se hizo en base a valores elevados de PSA, bien solicitado por el urólogo o por otro médico de Atención Primaria, frente a sólo el 13,4\% de la serie de 1995.

Respecto a las variables evaluadas del PSA, encontramos un valor medio del cociente de PSA libre/PSA total de $13,9 \%$ en la serie de pacientes de 2004. Considerado el punto de corte 
del 18\%, establecido por Christensson et al. ${ }^{7}$, cualquier porcentaje inferior al $18 \%$ discrimina significativamente mejor entre varones con y sin $\mathrm{CP}$, frente al uso exclusivo del PSA total como marcador aislado. Lo mismo ocurrió con la PSAV puesto que de los 92 pacientes en los que se pudo calcular este parámetro, tan sólo 16 presentaron unos valores inferiores a $0,75 \mathrm{ng} / \mathrm{ml} / \mathrm{año}$, punto de corte propuesto por Fang et al. ${ }^{8,9}$. Por lo que se refiere a la PSAD, su valor medio en el grupo de pacientes con concentraciones de PSA entre 4 y $10 \mathrm{ng} / \mathrm{ml}$ fue de $0,21 \mathrm{ng} / \mathrm{ml} / \mathrm{cc}$, superior al $0,15 \mathrm{ng} / \mathrm{ml} / \mathrm{cc}$ descrito por Catalona et al. ${ }^{10}$, con el que se detectarian el $95 \%$ de los tumores con una especificidad del $91 \%$.

La migración de la estadificación clínico patológica hacia estadios más precoces descrita tras el uso generalizado del PSA en pacientes asintomáticos ${ }^{4}$, se confirmó en nuestro estudio, como demuestra la mayor proporción de tumores de alto grado con Gleason entre 8-10 ng/ml diagnosticados en la serie del año 1995 y un mayor número de tumores de bajo grado en el año 2004. Se ha observado también una correlación entre la suma de Gleason y los niveles de PSA y tacto rectal, ya que se ha encontrado valores significativamente más altos del Score de Gleason en pacientes en donde el tacto rectal era sospechoso y la concentración de PSA mayor de $10 \mathrm{ng} / \mathrm{ml}$, sin embargo, en el momento actual no se puede realizar una preselección de pacientes con tumores clínicamente localizados, detectados mediante programas de cribado en los que el tratamiento radical ofrezca una mayor expectativa de vida que un programa de vigilancia. Actualmente se están llevando a cabo dos estudios europeos multicéntri- cos en los que se espera obtener resultados en el año 2007 que predigan los posibles beneficios de un programa de cribado para el CP.

\section{REFERENCIAS}

1. Herran Amo F. El cáncer de próstata en la comunidad de Madrid en el año 2000. Astrazeneca 2004;345-349.

2. Ferlay J, Bray F, Pissani P, Parkin DM. Globocan 2000: Cancer incidence, mortality and prevalence worldwide, version 1.0. LARC Cancerbase no. 5. Lyon: IARCPress: 2001.

3. Luján M, Páez A, Chiva V, Santonja C, Romero I, Berenguer A. Tendencias epidemiológicas en el cáncer de próstata durante los últimos años. Arch Esp Urol. 2004; 57(8): 817-825

4. Mettlin CJ, Murphy GP. Why is prostate cancer rate declining in the United States?. Cancer 1998; 82(2):229-245.

5. American Cancer Society. Cancer Facts \& Figures 2004. Atlanta, GA, 2004.

6. Herranz Amo F, Arias Fúnez F, Arrizabalaga Moreno M, Calahorra Fernández FJ, Carballido Rodríguez J, Diz Rodríguez R, et al. El cáncer de próstata en la Comunidad de Madrid en el año 2000. III- Estudio de extensión tumoral. Actas Urol Esp. 2003;27(3):411-417.

7. Christensson A, Bjork T, Nilsson. Serum prostate specific antigen complexed to alpha-antichymotrypsina as an indicator of prostate cancer. J Urol. 1993;150(11):100-103.

8. Fang J, Metter EJ, Landis P, Carter HO. PSA velocity for assessing prostate cancer risk in men with PSA levels between 2,0 and 4,0 ng/ml. Urology 2002;59(3):889-893.

9. Lynn NN, Collins GN, O`Reilly PH. The short term prostate specific antigen velocity before biopsy can be used to predict prostatic histology. BJU Int 2000;85(17):847-850.

10. Catalona WJ, Southwick PC, Slawin KM, Partin AW, Braver MK, Flanigan RC, et al. Comparisson of percent free PSA, PSA density and age-specific PSA cutoffs for prostate cancer detection and staging. Urology 2000;56(2):255-260.

Dr. M. Álvarez Múgica

Email: malvarez79@mixmail.com

(Trabajo recibido el 31 de julio de 2006) 